\title{
Single Nucleotide Polymorphism of the CYP2J2 Gene is Associated with Essential Hypertension in Uygur Population in China
}

\author{
Qing Zhu, Ali Amjad, Zhenyan Fu*, Yitong Ma, Ding Huang, Xiang Xie and Fen Liu
}

Department of Cardiovascular Medicine, First Affiliated Hospital of Xinjiang Medical University, Urumqi, China

\begin{abstract}
Background: Human Cytochrome P450 2J2 (CYP2J2) is the major arachidonic acid epoxygenase, which can metabolizes arachidonic acid (AA) to biologically active epoxyeicosatrienoic acids (EETs). The EETs are potent endogenous vasodilators and inhibitors of vascular inflammation. Recently, many evidence from models and human studies suggests that variability of CYP2J2 gene plays a mechanistic role in the development of hypertension. The aim of the present study was to assess the association between the human CYP2J2 gene polymorphism and Essential Hypertension $(E H)$ in a Han and Uygur population in China.
\end{abstract}

Methods: We used two independent case-control studies: a Han population (302 EH patients and 300 contro subjects) and a Uygur population (567 EH patients and 215 control subjects). All EH patients and controls were genotyped for the same three single nucleotide polymorphisms (SNPs) (rs890293, rs11572223 and rs2280275) of CYP2J2 gene by a Real-time PCR instrument.

Results: In the Uygur population, the distribution of SNP3 (rs2280275) genotypes, alleles and the dominant model (CC vs CT + TT) showed a significant difference between $\mathrm{EH}$ and control participants (for genotype: $\mathrm{P}=0.007$; for allele: $\mathrm{P}=0.001$; for dominant model: $\mathrm{P}=0.002$ ). The significant difference in dominant model was retained after adjustment for covariates (OR: $3.500,95 \%$ confidence interval $[\mathrm{Cl}]$ : $1.680-7.300, \mathrm{P}=0.001)$. However, all the above differences were not shown in the Han population.

Conclusions: The CC genotype of rs2280275 in CYP2J2 gene could be a risk genetic marker of EH and T allele may be a protective genetic marker of $\mathrm{EH}$ in Uygur population in China.

Keywords: CYP2J2; Single nucleotide polymorphism; Essential hypertension; Case-control study

\section{Introduction}

Essential hypertension (EH) is a complex multifactorial and polygenic disorder thought to result from an interaction between an individual's genetic makeup and different environments [1,2], which is a risk factor for other deadly diseases such as myocardial infarction and kidney failure [3-5]. It has been suggested that genetic variation may be a very important factor that could be responsible for $30 \%$ to $60 \%$ of inter-individual blood pressure (BP) variations [6]. However, the identity and function of the contributing loci are largely unknown.

CYP2J2, the single member of human cytochromes P450II J subfamily, is expressed at high levels in heart, predominantly in cardiac myocytes and endothelial cells of small and large coronary arteries and kidney [7]. It is responsible for not only the metabolism of xenobiotics but also a host of endogenous substance. In the human heart, CYP2J2 can mainly metabolize AA to biologically active epoxyeicosatrienoic acids (EETs), which play an important role in the regulation of cardiovascular inflammation [8], and possess potent vasodilatory, antiapoptotic properties in the cardiovascular system [9-11]. Common polymorphisms within CYP2J2 can result in the variation of EETs, which may determine susceptibility to the development of cardiovascular disease, such as hypertension [12] and coronary artery disease [9]. In animal model studies increased CYP2J2 expression was found in the spontaneous hypertension rat (SHR), with two-fold higher levels of 14, 15-EET and 11, 12-EET when compared to normotensive Wistar-Kyoto rats (WKY) [13,14]. And human studies also suggest that variability of CYP2J2 gene plays a mechanistic role in the development of hypertension $[12,15,16]$. However, these studies on the cardiovascular risk associated with CYP2J2 polymorphisms have provided inconsistent results. The studies by Sheng-Nan $\mathrm{Wu}$ and Alexey V. Polonikova showed a polymorphism of the CYP2J2 gene (rs890293) was associated with increased risk of EH $[15,16]$. By contrast, a decreased risk of $\mathrm{EH}$ was reported by Lorraine M. King in Caucasian males carrying CYP2J2 variant alleles (rs890293) [12] There was a study suggesting no significant association between the polymorphism of CYP2J2 (rs890293) and EH [17]. Based on the above study background, we aimed to assess the association between the polymorphism of CYP2J2 and EH in a Han and Uygur Population of China.

\section{Methods}

\section{Ethical approval of the study protocol}

Written informed consent was obtained from all participants. All participants explicitly provided permission for DNA analyses as well as collection of relevant clinical data. This study was approved by the Ethics Committee of the First Affiliated Hospital of Xinjiang Medical

*Corresponding author: Zhenyan $\mathrm{Fu}$, Department of Cardiovascula Medicine, First Affiliated Hospital of Xinjiang Medical University, Li Yu Shan South Road 137, Urumqi 830054, China, Tel: +86 991-4362611; Fax: +86 991-4366191; E-mail: fuzhenyan316@126.com

Received: November 05, 2014; Accepted: November 10, 2014; Published November 12, 2014

Citation: Zhu Q, Amjad A, Fu Z, Ma Y, Huang D, et al. (2015) Single Nucleotide Polymorphism of the CYP2J2 Gene is Associated with Essential Hypertension in Uygur Population in China. Biochem Anal Biochem 4: 159. doi: 10.4172/2161 1009.1000159

Copyright: (c) 2015 Zhu Q et al. This is an open-access article distributed unde the terms of the Creative Commons Attribution License, which permits unrestricted use, distribution, and reproduction in any medium, provided the original author and source are credited. 
University (Urumqi, China). It was conducted according to the standards of the Declaration of Helsinki.

\section{Subjects}

The subjects were from a Han population and a Uygur population who lived in the Xinjiang Uygur Autonomous Region of China. We recruited randomly 302 Han patients and 567 Uygur patients with EH and 300 and 215 ethnically and geographically matched control groups in First Affiliated Hospital of Xinjiang Medical University from 2006 to 2013. A total of 869 patients who consistently had systolic blood pressures $(\mathrm{SBP}) \geq 140 \mathrm{mmHg}$ and/or and diastolic blood pressures (DBP) $\geq 90 \mathrm{mmHg}$ were diagnosed as being hypertensive [18]. And all $\mathrm{EH}$ subjects investigated in the present study had to have a family history of $\mathrm{EH}$, with a family history of hypertension defined as a prior diagnosis of hypertension in the grandparents, parents, uncles, aunts, or siblings and exclude any subjects with secondary hypertension, such as primary aldosteronism or kidney disease. All 365 control groups had $\mathrm{SBP}<130 \mathrm{mmHg}$ and $\mathrm{DBP}<80 \mathrm{mmHg}$ simultaneously.

\section{Blood collection and DNA extraction}

Fasting Blood samples were drawn by venepuncture in the catheterroom from all participants. The blood samples were drawn into a 5 $\mathrm{ml}$ ethylene diamine tetraacetic acid (EDTA) tube and centrifuged at $4000 \times \mathrm{g}$ for $5 \mathrm{~min}$ to separate the plasma content. Genomic DNA was extracted from the peripheral leukocytes using standard phenolchloroform method. The DNA samples were stored at $-80^{\circ} \mathrm{C}$ until use. While used, the DNA was diluted to $50 \mathrm{ng} / \mu \mathrm{L}$ concentration.

\section{Genotyping}

There are 701 SNPs for the human CYP2J2 gene listed in the National Center for Biotechnology Information SNP database (http:// www.ncbi.nlm.nih.gov/SNP). Using the Haploview 4.2 software and the HapMap phrase II database, we obtained three tag SNPs (rs890293, rs11572223 and rs2280275) by using minor allele frequency (MAF) $\leq 0.1$ and linkage disequilibrium patterns with $\mathrm{r}^{2} \geq 0.5$ as a cut off. The position of the SNP1, SNP2 and SNP3 (rs890293, rs11572223 and rs2280275) was by order of increasing distance from the CYP2J2 gene 5 'end (Figure 1). SNP1 (rs890293) was observed in the proximal promoter region of the gene. The polymorphisms caused a loss of transcription factor binding site Sp1. Genotyping was undertaken using the TaqMan ${ }^{\star}$ SNP Genotyping Assay (Applied Bio systems). The primers and probes used in the TaqMan ${ }^{\circ}$ SNP Genotyping Assays (ABI) were chosen based on information at the ABI website (http:// myscience.appliedbiosystems.com). Thermal cycling was done using the Applied Biosystems7900HT Standard Real-Time PCR System. Plates were read on Sequence Detection Systems (SDS) automation controller software v2.3 (ABI).PCR amplification was performed using $3.0 \mu \mathrm{l}$ of TaqMan Universal Master Mix, $0.15 \mu \mathrm{l}$ probes and $1.85 \mathrm{dd}$ $\mathrm{H}_{2} \mathrm{O}$ in a $6-\mu \mathrm{l}$ final reaction volume containing $1 \mu \mathrm{l}$ DNA.Thermal cycling conditions were as follows: $95^{\circ} \mathrm{C}$ for $5 \mathrm{~min} ; 40$ cycles of $95^{\circ} \mathrm{C}$ for $15 \mathrm{~s}$; and $60^{\circ} \mathrm{C}$ for $1 \mathrm{~min}$. All 96 wells Plates were read on Sequence Detection Systems (SDS) automation controller software v2.3 (ABI).

\section{Biochemical analysis}

Serum concentrations of Total Cholesterol (TC), Triglyceride (TG), Glucose (Glu), High-Density Lipoprotein Cholesterol (HDL-C), Low-Density Lipoprotein Cholesterol (LDL-C), were measured using standard methods in the Central Laboratory of First Affiliated Hospital of Xinjiang Medical University.

\section{Statistical analysis}

All continuous variables (e.g. age, Body Mass Index: BMI, pulse, cholesterol levels) are presented as means \pm standard deviation (S.D.). The difference between the $\mathrm{EH}$ and Control groups was analyzed using an independent-sample T-Test. The differences in the frequencies of smoking, Diabetes Mellitus (DM), and CYP2J2 genotypes were analyzed using $\chi^{2}$ test or Fisher's exact test while appropriate. HardyWeinberg equilibrium was assessed by $\chi^{2}$ analysis. Logistic regression analyses with effect ratios (odds ratio [OR] and 95\% CI) were used to assess the contribution of the major risk factors. All statistical analyses were performed using SPSS 17.0 for Windows (SPSS Institute, Chicago, USA). P-values of less than 0.05 were considered to statistically significant.

\section{Results}

Table 1 shows the clinical characteristics of the study participants. For Han and Uygur population, there was no significant difference in age between $\mathrm{EH}$ patients and control subjects it means the study was agematched case-control study. In Han population, BMI was significantly higher for patients with $\mathrm{EH}$ than for control participants, and the rest of the indicators were no difference between $\mathrm{EH}$ patients and control subjects. In Uygur population, BMI, the plasma concentration of LDL-C and the prevalence of DM were significantly higher for patients with $\mathrm{EH}$ than for control participants. And the rest of the indicators were no difference between $\mathrm{EH}$ patients and control subjects.

Table 2 shows the distribution of genotypes and alleles of SNP1, SNP2 and SNP3 for the CYP2J2 gene. In the Uygur population, the distribution of SNP3 (rs2280275) genotypes, alleles and the dominant model (CC vs CT+TT) showed a significant difference between $\mathrm{EH}$ and control participants (for genotype: $\mathrm{P}=0.007$; for allele: $\mathrm{P}=0.001$; for dominant model: $\mathrm{P}=0.002$ ). $\mathrm{T}$ allele of $\mathrm{rs} 2280275$ was significantly lower in $\mathrm{EH}$ patients than in control participants (14.50\% vs $21.20 \%)$. The dominant model (CC vs CT+TT) of rs2280275 was significantly higher in $\mathrm{EH}$ patients than in control participants (74.60\% vs $63.30 \%)$. However, all the above differences were not shown in the Han population.

Table 3 shows that multiple logistic regression analyses were done with Glu, LDL-C, DM, and smoking because these variables were the major confounding factors for $\mathrm{EH}$. The significant difference of rs2280275 was retained after adjustment for Glu, LDL-C, DM, and smoking in Uygur population (OR: $3.500,95 \%$ confidence interval [CI]: 1.680-7.300, $\mathrm{P}=0.001)$.

\section{Discussion}

Several CYP enzyme families have been identified in the heart, endothelium and smooth muscle of blood vessels. In humans, CYP2J2 acts mainly converting arachidonic acid to EETs, and EETs have been proposed to regulate vascular tone and fluid-electrolyte transport in cardiovascular and renal tissues, suggesting a crucial role of epoxygenase-derived eicosanoids in blood pressure regulation $[19,20]$. To demonstrate the involvement of these CYP2J2-derived eicosanoids in the pathogenesis of $\mathrm{EH}$, a number of literature data were summarized in Figure 2. 1) In the vascular cells, EETs activate $\mathrm{Ca}^{2+}$-dependent $\mathrm{K}^{+}$channels and $\mathrm{Na}-\mathrm{K}$-ATPase resulting to the hyperpolarization of cell membranes and vasorelaxation [21]. 2) EETs increase intracellular $\mathrm{Ca}^{2+}$ concentration in endothelial cells stimulating the formation and release of nitric oxide, prostaglandins (PGI, PGE2, and PGF2 $\alpha$ ), thromboxane and other vasoactive substances [22]. 3) In the kidney, EETs are important regulators of glomerular filtration by activating 
Citation: Zhu Q, Amjad A, Fu Z, Ma Y, Huang D, et al. (2015) Single Nucleotide Polymorphism of the CYP2J2 Gene is Associated with Essential Hypertension in Uygur Population in China. Biochem Anal Biochem 4: 159. doi: 10.4172/2161-1009.1000159

Page 3 of 6

\begin{tabular}{|c|c|c|c|c|c|c|}
\hline & \multicolumn{3}{|c|}{ Han } & \multicolumn{3}{|c|}{ Uygur } \\
\hline & EH patients & Control subjects & $p$ Value & EH patients & Control subjects & $p$ Value \\
\hline Number (n) & 302 & 300 & & 567 & 215 & \\
\hline Age (years) & $57.97 \pm 9.87$ & $56.26 \pm 10.43$ & 0.159 & $53.33 \pm 8.97$ & $54.74 \pm 8.41$ & 0.74 \\
\hline BMI $\left(\mathrm{kg} / \mathrm{m}^{2}\right)$ & $26.42 \pm 3.20$ & $25.09 \pm 3.21$ & $0.001^{*}$ & $30.98 \pm 6.61$ & $26.99 \pm 3.59$ & $0.0001^{*}$ \\
\hline Pulse (beats/min) & $73.62 \pm 10.10$ & $73.47 \pm 9.69$ & 0.897 & $77.47 \pm 10.21$ & $74.71 \pm 10.06$ & 0.102 \\
\hline Glu (mmol/L) & $5.63 \pm 1.55$ & $5.47 \pm 1.49$ & 0.365 & $5.30 \pm 1.48$ & $5.31 \pm 1.04$ & 0.932 \\
\hline $\mathrm{TG}(\mathrm{mmol} / \mathrm{L})$ & $1.88 \pm 1.19$ & $1.83 \pm 1.35$ & 0.770 & $1.71 \pm 1.02$ & $1.78 \pm 1.00$ & 0.37 \\
\hline $\mathrm{TC}(\mathrm{mmol} / \mathrm{L})$ & $4.41 \pm 0.96$ & $4.25 \pm 0.95$ & 0.164 & $4.06 \pm 0.97$ & $4.12 \pm 1.15$ & 0.421 \\
\hline $\mathrm{HDL}(\mathrm{mmol} / \mathrm{L})$ & $1.17 \pm 0.29$ & $1.13 \pm 0.34$ & 0.175 & $1.01 \pm 0.22$ & $1.02 \pm 0.45$ & 0.542 \\
\hline $\mathrm{LDL}(\mathrm{mmol} / \mathrm{L})$ & $2.44 \pm 0.86$ & $2.40 \pm 0.82$ & 0.777 & $2.69 \pm 0.85$ & $2.48 \pm 0.87$ & $0.003^{*}$ \\
\hline DM (\%) & 14.6 & 11.3 & 0.493 & 81.89 & 20.5 & $0.0001^{*}$ \\
\hline Smoke (\%) & 39.7 & 43.3 & 0.560 & 56.9 & 50.3 & 0.182 \\
\hline
\end{tabular}

Table 1: The clinical characteristics of the study participants.

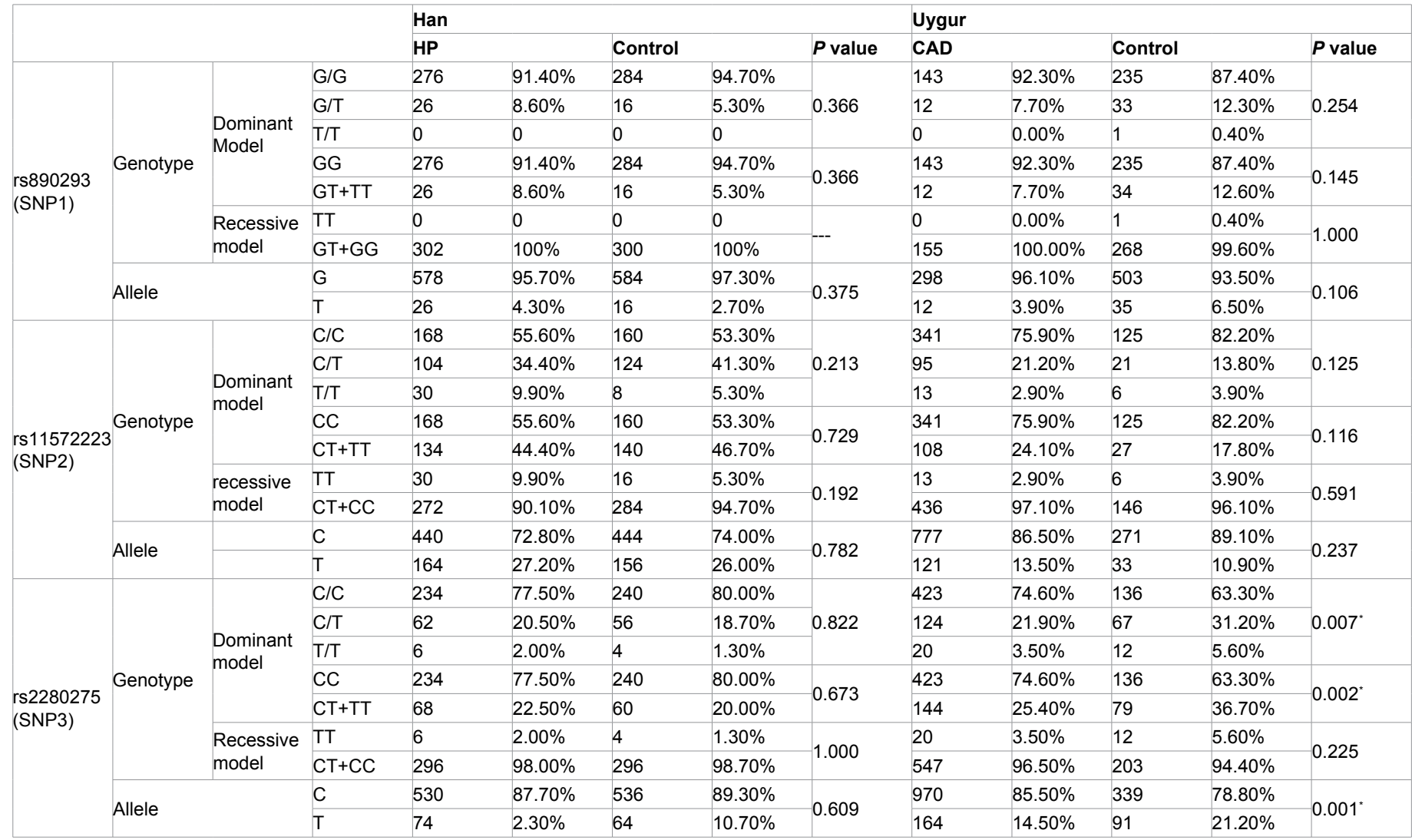

Table 2: Genotype and allele distributions in patients with $C A D$ and control subjects.

\begin{tabular}{|c|c|c|c|}
\hline & OR & $\mathbf{9 5 \%}$ & P \\
\hline Dominant model(CC vs CT +TT) & 3.500 & $1.68-7.30$ & $0.001^{*}$ \\
\hline age & 1.011 & $0.972-1.053$ & 0.584 \\
\hline BMI & 1.097 & $1.011-1.190$ & 0.026 \\
\hline Glu & 1.057 & $0.834-1.340$ & 0.214 \\
\hline TG & 1.121 & $0.719-1.748$ & 0.614 \\
\hline TC & 0.722 & $0.360-1.448$ & 0.359 \\
\hline HDL-C & 0.624 & $0.238-1.633$ & 0.336 \\
\hline LDL-C & 1.522 & $0.665-3.482$ & 0.320 \\
\hline DM & 27.037 & $12.372-59.085$ & $0.0001^{*}$ \\
\hline
\end{tabular}

Table 3: Multiple logistic regression analysis for CAD patients and control subjects of Uygur population. 
Citation: Zhu Q, Amjad A, Fu Z, Ma Y, Huang D, et al. (2015) Single Nucleotide Polymorphism of the CYP2J2 Gene is Associated with Essential Hypertension in Uygur Population in China. Biochem Anal Biochem 4: 159. doi: 10.4172/2161-1009.1000159

CYP2J2 gene

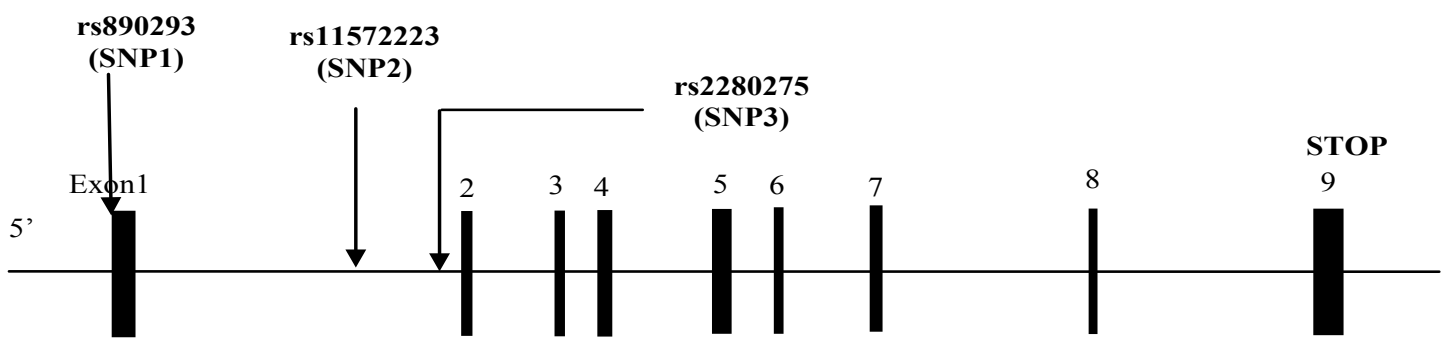

o (kbp)

10

20

1

30

40

Figure 1: Structure of the human CYP2J2 gene. This gene consists of 9 exons separated by 8 introns. Boxes indicate exons, and lines indicate introns and intergenic regions. Filled boxes indicate coding regions. Arrows mark the locations of polymorphisms.

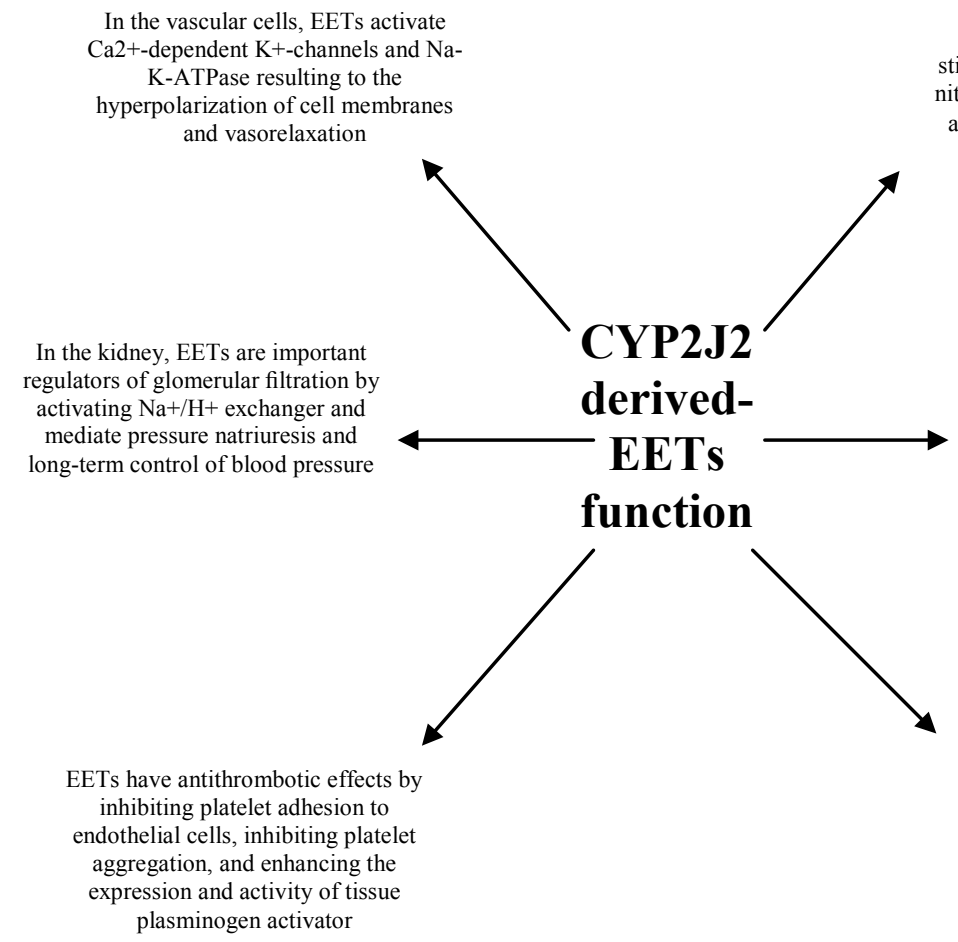

In the vascular cells, EETs activate

K-ATPase resulting to the of cell membranes

EETS

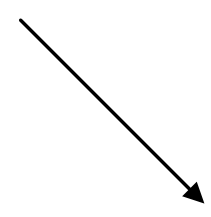

EETs increase intracellular $\mathrm{Ca} 2+$

concentration in endothelial cells stimulating the formation and release of nitric oxide, prostaglandins (PGI, PGE2, and PGF2 $\alpha$ ), thromboxane and other vasoactive substances
EETs inhabit inflammation responses by decreasing the cytokine-induced endothelial expression of vascular cell adhesion molecule-1 (VCAM-1) and to decrease leukocytes adhesion to the vascular wall by inhibiting nuclear factor $\kappa \mathrm{B}(\mathrm{NF}-\kappa \mathrm{B})$ and $\mathrm{I} \kappa \mathrm{B}$ kinase

Figure 2: Schematic of EET interactions with cardiovascularion channels.

$\mathrm{Na}^{+} / \mathrm{H}^{+}$exchanger and mediate pressure natriuresis and long-term control of blood pressure [23,24]. 4) EETs inhabit inflammation responses by decreasing the cytokine-induced endothelial expression of vascular cell adhesion molecule-1 (VCAM-1) and to decrease leukocytes adhesion to the vascular wall by inhibiting nuclear factor $\kappa \mathrm{B}(\mathrm{NF}-\kappa \mathrm{B})$ and IкB kinase [25]; 5) EETs have antithrombotic effects by inhibiting platelet adhesion to endothelial cells, inhibiting platelet aggregation, and enhancing the expression and activity of tissue plasminogen activator [26]; 6) EETs have been suggested to increase the proliferation of various cell types, including vascular smooth muscle cells and endothelial cells demonstrating a potential role of cytochrome P450 eicosanoids to promote vascular remodeling [27]. Recent studies suggest that human CYP2J2 gene is highly polymorphic [28], and it has been proposed that genetic polymorphisms within the gene might contribute to expression and/or activity of the enzyme and affect the metabolism of arachidonic acid, resulting in an altered synthesis of EETs. In this study, we hypothesized that variability in the gene might affect the risk of EH. We genotyped three SNPs of the 
gene in a Han population and a Uygur population, and assessed the association between the polymorphism of CYP2J2 gene and EH using case-control analyses.

In our case-control study, we found a significant difference in the genotype frequency of rs2280275, an intronic polymorphism, between hypertensive group and control group in the Uygur population. In particular, we observed that being a CC homozygote for rs 2280275 was a risk factor for $\mathrm{EH}$ in Uygur population. Although rs2280275 is an intronic polymorphism, it is unlikely to cause direct changes of amino acids. Owing to its location close to the splice site of exon2 of CYP2J2, it may be a promising candidate variant that could change the dimensional structure of DNA, influencing splicing and transcription. Splicing variants have been described as potential factors for inducing variable gene expression. Two good examples of this is the intronic polymorphism CYP3A5*3 and rs1155002 of CYP2J2 that activate alternative splicing and lead to the most frequent defect of CYP3A5 expression and overexpression of CYP2J2 [15,29].

However, in the Han population, there was no difference in the genotype and allele frequency of rs2280275 between hypertensive group and control group. This difference between Uygur population and Han population was not only the racial differences and genetic differences, but also the different life style of the two populations. Uygurs diet was with more red meat, dairy and pasta, fewer fruits and vegetables, and showing the diets features of high-fat, high-protein, high-salt and high-glucose. In addition, Uygur population comparing to Han population has a different life style with smoking, sedentary life style, low socio economic and literacy status and stressful life style. Studies have shown that Uygurs were more likely to suffer from coronary heart disease, diabetes and hypertension. As expected, the Uygur patients have significant higher rate of DM and smoking which was not observed in the Han patients in our study (Table 1). Diabetes and smoking both are the risk factors for hypertension. This may cause the differences between the two populations.

Though SNP1 (rs890293) was observed in the proximal promoter region of the CYP2J2 gene, and the polymorphisms caused a loss of transcription factor binding site Sp1, resulting in the synthesis of EETs was reduced. The studies about the association between CYP2J2 polymorphisms (rs890293) and the cardiovascular risk have provided inconsistent results. The study by Wu Shengnan et al. showed a functionally relevant polymorphism of the CYP2J2 gene (rs890293) was associated with an increased risk of Essential hypertension [15]. This result was supported by Polonikova AV et al. the study of showing the polymorphism of CYP2J2 (rs890293) was an important risk factor for the development of EH in Chinese [16]. In addition, a decreased risk of EH was reported by King Lorraine et al. in Caucasians males carrying CYP2J2 variant alleles (rs890293), but no significant association was observed in African-Americans [12]. Our study was consistent with the study of Dreisbach [17]. showing no significant association between the polymorphism of CYP2J2 (rs890293) and EH. These reasons to explain some differences might include: 1) a difference in the genetic backgrounds between populations studied; 2) confounding by population stratification within ethnically heterogeneous; 3 ) chance or artifact, and possibly 4) due to yet unspecified environmental or genetic factors in populations studied. For the SNP2 (rs11572223), our result showed that the genotype and allele distributions were not different between the EH patients and control subjects.

\section{Conclusion}

In conclusion, we found that rs2280275 may be a novel polymorphism of the CYP2J2 gene associated with $\mathrm{EH}$ in Uygur population in China. The CC genotype of rs2280275 in CYP2J2 gene could be a risk genetic marker of $\mathrm{EH}$ and $\mathrm{T}$ allele may be a protective genetic marker of EH in Uygur population in China. Certainly it needs a large number of clinical samples to study further in China.

\section{Acknowledgement}

This work was supported by the grants from National Natural Science Foundation of China (81060020) and (81260041). Qing Zhu, Ali Amjad -These authors contributed equally to this work.

\section{References}

1. Manace LC, Godiwala TN, Babyatsky MW (2009) Genomics of cardiovascular disease. Mt Sinai J Med 76: 613-623.

2. Frazier L, Johnson RL, Sparks E (2005) Genomics and cardiovascular disease J Nurs Scholarsh 37: 315-321.

3. Fu C, Wang H, Wang S, Shi Y, Zhou X, et al. (2008) Association of beta 1-adrenergic receptor gene polymorphisms with left ventricular hypertrophy in human essential hypertension. Clin Biochem 41: 773-778.

4. Tseng $\mathrm{CH}$, Tseng CP, Chong $\mathrm{CK}$ (2010) Joint effects of hypertension, smoking dyslipidemia and obesity and angiotensin-converting enzyme DD genotype on albuminuria in Taiwanese patients with type 2 diabetes mellitus. Clin Biochem; 43:629-634.

5. Huang SS, Huang PH, Chiang KH, Chen JW, Lin SJ (2011) Association of serum bilirubin levels with albuminuria in patients with essential hypertension. Clin Biochem 44: 859-863.

6. Binder A (2007) A review of the genetics of essential hypertension. Curr Opin Cardiol 22: 176-184

7. Wu S, Moomaw CR, Tomer KB, Falck JR, Zeldin DC (1996) Molecular cloning and expression of CYP2J2, a human cytochrome P450 arachidonic acid epoxygenase highly expressed in heart. J Biol Chem 271: 3460-3468.

8. Moshal KS, Zeldin DC, Sithu SD, Sen U, Tyagi N, et al. (2008) Cytochrome P450 (CYP) 2J2 gene transfection attenuates MMP-9 via inhibition of NFkappabeta in hyperhomocysteinemia. J Cell Physiol 215: 771-781.

9. Spiecker M, Darius H, Hankeln T, Soufi M, Sattler AM, et al. (2004) Risk of coronary artery disease associated with polymorphism of the cytochrome P450 epoxygenase CYP2J2. Circulation 110: 2132-2136.

10. Fisslthaler B, Popp R, Kiss L, Potente M, Harder DR, et al. (1999) Cytochrome P450 2C is an EDHF synthase in coronary arteries. Nature 401: 493-497.

11. Spiecker M, Liao J (2006) Cytochrome P450 epoxygenase CYP2J2 and the risk of coronary artery disease. Trends Cardiovasc Med 16: 204-208.

12. King LM, Gainer JV, David GL, Dai D, Goldstein JA, et al. (2005) Single nucleotide polymorphisms in the CYP2J2 and CYP2C8 genes and the risk of hypertension. Pharmacogenet Genomics 15: 7-13.

13. Yu Z, Huse LM, Adler P, Graham L, Ma J, et al. (2000) Increased CYP2 expression and epoxyeicosatrienoic acid formation in spontaneously hypertensive rat kidney. Mol Pharmacol 57: 1011-1020.

14. Xiao B, Li X, Yan J, Yu X, Yang G, et al. (2010) Overexpression of cytochrome P450 epoxygenases prevents development of hypertension in spontaneously hypertensive rats by enhancing atrial natriuretic peptide. J Pharmacol Exp The 334: 784-794.

15. Wu SN, Zhang Y, Gardner CO, Chen $Q$, Li Y et al. (2007) Evidence for association of polymorphisms in CYP2J2 and susceptibility to essential hypertension. Ann Hum Genet 71: 519-525.

16. Polonikov AV, Ivanov VP, Solodilova MA, Khoroshaya IV, Kozhuhov MA, et al. (2008) A common polymorphism G-50T in cytochrome P450 2J2 gene is associated with increased risk of essential hypertension in a Russian population. Dis Markers 24: 119-126.

17. Dreisbach AW, Japa S, Sigel A, Parenti MB, Hess AE, et al. (2005) The Prevalence of $\mathrm{CYP} 2 \mathrm{C} 8,2 \mathrm{C} 9,2 \mathrm{~J} 2$, and soluble epoxide hydrolase polymorphisms in African Americans with hypertension. Am J Hypertens 18 1276-1281.

18. Guidelines Subcommittee: 1999 World Health Organization-International Society of Hypertension Guidelines for the Management of Hypertension. Hypertension; 17:151-833. 
Citation: Zhu Q, Amjad A, Fu Z, Ma Y, Huang D, et al. (2015) Single Nucleotide Polymorphism of the CYP2J2 Gene is Associated with Essential Hypertension in Uygur Population in China. Biochem Anal Biochem 4: 159. doi: 10.4172/2161-1009.1000159

19. Campbell WB, Harder DR (1999) Endothelium-derived hyperpolarizing factors and vascular cytochrome $\mathrm{P} 450$ metabolites of arachidonic acid in the regulation of tone. Circ Res 84: 484-488.

20. Zeldin DC (2001) Epoxygenase pathways of arachidonic acid metabolism. J Biol Chem 276: 36059-36062.

21. Fleming I (2001) Cytochrome p450 and vascular homeostasis. Circ Res 89 753-762.

22. Roman RJ (2002) P-450 metabolites of arachidonic acid in the control of cardiovascular function. Physiol Rev 82: 131-185.

23. Harris RC, Munger KA, Badr KF, Takahashi K (1990) Mediation of renal vascular effects of epidermal growth factor by arachidonate metabolites. FASEB J 4: 1654-1660.

24. Dos Santos EA, Dahly-Vernon AJ, Hoagland KM, Roman RJ (2004) Inhibition of the formation of EETs and 20-HETE with 1-aminobenzotriazole attenuates pressure natriuresis. Am J Physiol Regul Integr Comp Physiol 287: R58-68.
25. Node K, Huo Y, Ruan X, Yang B, Spiecker M, et al. (1999) Anti-inflammatory properties of cytochrome P450 epoxygenase-derived eicosanoids. Science 285: $1276-1279$

26. Jiang H, McGiff JC, Quilley J, Sacerdoti D, Reddy LM, et al. (2004) Identification of 5,6-trans-epoxyeicosatrienoic acid in the phospholipids of red blood cells. $J$ Biol Chem 279: 36412-36418

27. Uddin MR, Muthalif MM, Karzoun NA, Benter IF, Malik KU (1998) Cytochrome P-450 metabolites mediate norepinephrine-induced mitogenic signaling Hypertension 31: 242-247.

28. Spiecker M, Darius H, Hankeln T, Soufi M, Sattler AM, et al. (2004) Risk of coronary artery disease associated with polymorphism of the cytochrome $\mathrm{P} 450$ epoxygenase CYP2J2. Circulation 110: 2132-2136.

29. Kuehl P, Zhang J, Lin Y, Lamba J, Assem M, et al. (2001) Sequence diversity in CYP3A promoters and characterization of the genetic basis of polymorphic CYP3A5 expression. Nat Genet 27: 383-391. 